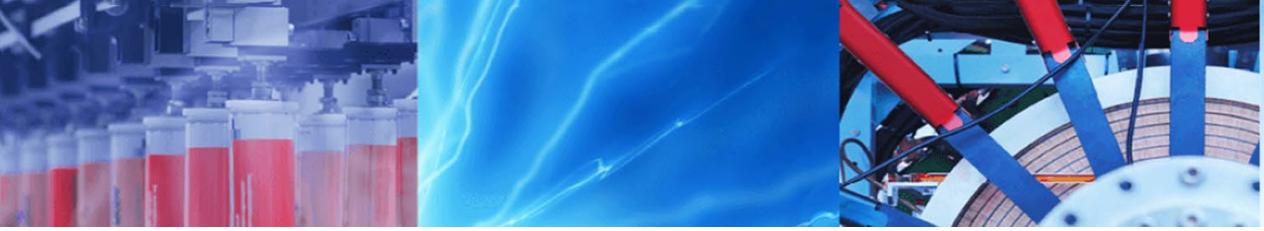

Research Article

\title{
Preparation of branched polyurethane curing agent by condensation polymerization and radical polymerization
}

\author{
Wang Chuanxing ${ }^{1} \cdot$ Wang Ning $^{1} \cdot$ Wang Jing $^{1} \cdot$ Hou Baorong ${ }^{1}$
}

Received: 6 May 2020 / Accepted: 4 November 2020 / Published online: 21 November 2020

(c) Springer Nature Switzerland AG 2020

\begin{abstract}
A new PU curing agent with branched structure was prepared by esterification, radical polymerization and reaction of TDI. Unsaturated content (itaconic acid) was added in esterification of adipic acid and 1,4-butanediol, which prepared linear molecule poly (itaconic acid-adipic acid-1,4-butanediol). Then, azodiisobutyronitrile was used to initiate polymerization to form branched molecule (CPBIA). CPBIA reacted with semi-blocked TDI to prepare TDI-CPBIA. These polymers were characterized by FT-IR, ${ }^{1} \mathrm{H}$ NRM and GPC. Their structure was confirmed. The adjustment of polycondensation and radical polymerization by the raw material ratio and reaction temperature could yield the desired $\mathrm{PU}$ curing agent with branched structure and fine solubility. According primary experiments, TDI-CPBIA had fine application performance. The coating had good hardness $(6 \mathrm{H})$, thermal performance $\left(\mathrm{T}_{0}>250^{\circ} \mathrm{C}\right)$, adhesion (level 1$)$ and impact strength $(>50 \mathrm{~cm})$. This PU curing agent has fine application performance.
\end{abstract}

Keywords Polyurethane $\cdot$ Branched polymer $\cdot$ PU curing agent

\section{Introduction}

The demand for eco-friendly coatings, like waterborne coatings [1, 2], high solid coatings [3], UV curable coatings [4,5] and powder coatings [6], has increased rapidly. Environmental concerns were the major factors behind this shift, such as recycling or waste disposal at the end of their life circle, volatile organic solvent emissions.

Polyurethane coatings had become the mainstream coatings for industrial coatings and civilian coatings because of their advantages such as good appearance, high hardness, abrasion resistance, wide applicable temperature and low cost [7]. Curing agent was a very important component in the coating system. The usual curing agents included toluene diisocyanate (TDI) trimer [8], 4,4'-methylenediphenyl diisocyanate (MDI) $[9,10]$, hexamethylene diisocyanate (HDI) [11] and isophorone diisocyanate (IPDI) [12]. Diphenylmethane diisocyanate (MDI) was used as curing agent for its low toxicity $[13,14]$. For its symmetry of MDI molecular structure, the strength, wear resistance and elasticity of coating film prepared by MDI were better than that of TDI. It was found by Yao Xiao et al. [10] that MDI-50-based waterborne polyurethane showed better properties compared to IPDI based waterborne polyurethane. Davletbaeva, I. M. found that segmented polyurethanes with the predetermined length of the rigid block were formed in the case of 4,4'-MDI, while the use of polyisocyanate gave rise to framed aromatic polyurethanes [15]. Though there were many kinds of the PU curing agents, TDI and its derivatives were the most popular.

However, free toluene diisocyanate (TDI) was often contained in the curing agent of polyurethane coatings, which has a great influence on the environment [16].

TDI-TMP prepolymer was prepared with TDI and trimethylolpropane (TMP) at certain condition [17]. It was the most common polyurethane curing agent and one of the earliest isocyanate prepolymer components [17]. There was a problem of free TDI in the curing

Wang Ning, 39870019@qq.com | 'Institute of Oceanology, Chinese Academy of Sciences, Qingdao 266071, Shandong, China. 
agent because TDI was toxic $[18,19]$. There were many researches on how to reduce the content of free TDI in TDI prepolymer, which were summarized as follows: molecular sieve adsorption [20], extraction [21, 22], chemical reaction [23], thin film evaporation $[24,25]$ and azeotropic distillation [26].

Molecular sieve was silicate crystals by artificial hydrothermal synthesis with highly efficient, deep and selective adsorbents, so it had high separation precision and separation rate [27]. Marans [20] used X type zeolite molecular sieve to adsorb free TDI in the tower. The prepolymer was put into the molecular sieve tower and made contact to promote the absorption of free TDI. The temperature of the prepolymer entering the tower was about $20 \sim 100{ }^{\circ} \mathrm{C}$. The temperature of the tower was $40 \sim 85^{\circ} \mathrm{C}$. The temperature of molecular sieve and prepolymer was $50 \sim 75^{\circ} \mathrm{C}$. Then, molecular sieve was removed by filtration or centrifugation. TDI content of the prepolymer was greatly reduced. This method also avoided the possibility of reducing the effective NCO in the prepolymer by high temperature heating in the thin film evaporator. This process could reduce the mass fraction of TDI in the prepolymer by $50 \sim 99 \%$.

The extraction method was based that the higher molecular weight fraction of the reaction product was soluble in the strong solvent, while the unreacted free TDI was only soluble in hydrocarbon or naphthenic hydrocarbon solvents, such as gasoline, petroleum ether, hexane, cyclohexane, benzene or its mixture. The extraction method included supercritical fluid extraction and ordinary solvent extraction. Robin [22] used carbon dioxide (or ethane, propane, butane and ethylene) as extractant. The mass fraction of isocyanate monomer in prepolymer was reduced to $0.03 \%$, and the mass fraction of isocyanate dimers was reduced to less than $1 \%$. Zhang Jie [28] used petroleum ether as the extractant and continuous circulation extraction, but the result was not very ideal. However, the separation effect was better with the azeotropic distillation.

Chemical reaction method could be divided into two kinds, the direct synthesis method and the catalytic polymerization method. The principle of catalytic polymerization was to add small molecule hydroxyl compounds that could react with TDI monomer and promote the polymerization of TDI-TMP in the prepolymer, so that TDI could be transformed into small molecular carbamate or TDI polymer. The content of free TDI was reduced to $0.2 \sim 0.3 \%$ [29]. In USP3384624 [30], Benzyl alcohol and benzyl alcohol derivatives with various hindrance groups on the benzene ring were added to the prepolymer, which could react with free TDI under heating to reduce free TDI content. Benzene ring and the hindrance groups on benzene ring could inhibit the reaction between benzyl alcohol and NCO group of prepolymer, but could react with NCO group of TDI.

Thin film evaporation was a method of removing free TDI from curing agent under the condition of high vacuum by using outer cooled or internally cooled thin-film evaporator. The low free TDI product has been widely used [17]. The characteristics of high vacuum thin film evaporation are products with low molecular weight, narrow molecular weight distribution, high NCO content and high crosslink density. In USP3183112 [31], the prepolymer first heated the flash and then went into the thin film evaporator to remove the TDI. At that time, the concentration of free TDI was reduced to less than $2 \%$. In several patents [32, 33], free TDI was removed by thin film evaporator at $100-160^{\circ} \mathrm{C}$ and $0.1 \sim 0.8 \mathrm{~mm} \mathrm{Hg}$. In USP 4,385,171, azeotropic distillation was used to remove free monomer. Diisocyanate content was lower than $0.05 \%$ [26].

TDI-TMP was general PU curing agent. In addition, news polymer with branched chain could be prepared by esterification, radical polymerization and reaction of $-\mathrm{OH}$ and -NCO of TDI. News PU curing agent had several advantages such as low toxic, low viscosity and good application properties.

\section{Experiments}

\subsection{Materials}

Itaconic acid (IA, industrial product) was purchased from Qingdao Langyatai Group Co., China. Other reagents were (analytical reagent, AR) purchased from Sinopharm chemical reagent Co., Ltd., such as adipic acid, 1,4-butanediol, tetrabutyl titanate, hydroquinone, methyl alcohol, azodiisobutyronitrile, acetone, o-chlorophenol, toluene diisocyanate (TDI) and dibutyltin dilaurate.

\subsection{Preparation of polyester}

$\mathrm{IA}$, adipic acid and 1,4-butanediol esterified in four-neck flask with tetrabutyl titanate as catalyst and hydroquinone as polymerization inhibitor. Reaction conditions was the temperature $170{ }^{\circ} \mathrm{C}$, reaction time $6 \mathrm{~h}$, the mole ratio of $-\mathrm{OH}$ and $-\mathrm{COOH}$ 1.2:1. The sample was dissolved into the methanol. Then, unreacted content was removed by water extraction. The product was dried in the oven at $80^{\circ} \mathrm{C}$.

\subsection{Polyester crosslinking}

Polyester polymerized in 4-neck flask with toluene as solvent, azodiisobutyronitrile as initiator under the condition of nitrogen atmosphere, reaction temperature $80^{\circ} \mathrm{C}$, reaction time $5 \mathrm{~h}$. The sample was dried for $4 \mathrm{~h}$. 


\subsection{Preparation of PU curing agent}

TDI and o-chlorophenol were added into a 4-neck flask at room temperature and nitrogen atmosphere with the mole ratio of 1:1.2 (TDI: o-chlorophenol). After $0.5 \mathrm{~h}$, the system was heated to $90^{\circ} \mathrm{C}$ and kept for $5 \mathrm{~h}$. Semi-blocked TDI was prepared.

Semi-blocked TDI solution with acetone as the solvent was put into constant pressure drop funnel. It was dropped slowly into crosslinking polyester with dibutyltin dilaurate as the catalyst at $40^{\circ} \mathrm{C}$ and nitrogen atmosphere for $2.5 \mathrm{~h}$. The sample was dried at $40^{\circ} \mathrm{C}$ for $4 \mathrm{~h}$.

\subsection{Characterization}

Fourier transform infrared (FT-IR) spectra were recorded with NICOLET MAGNA-IR750 Series II from 4000 to $600 \mathrm{~cm}^{-1}$.

${ }^{1} \mathrm{H}$ NMR spectra were recorded with a Bruker $300 \mathrm{MHz}$ spectrometer at room temperature using DMSO- $\mathrm{d} 6$ as the solvent.

The molecular weights were measured by gel permeation chromatography (GPC, Shodex KF-805) using a liquid chromatograph equipped with TSK gel Super Mutipore $\mathrm{HZ}-\mathrm{M} * 2$, Flow rate $0.35 \mathrm{ml} / \mathrm{min}$, the concentration $1.0 \mathrm{mg} /$ $\mathrm{ml}$, injection volume $20 \mu \mathrm{l}$ and column temperature $40^{\circ} \mathrm{C}$. Tetrahydrofuran (THF) was used as the eluent. The calibration was polystyrene $\left(M_{w} 750-570 \times 10^{4}\right)$.

The acid value of polymer was tested according to ASTM D465-01. The acetylating reagent method (IS 354, Part-1, 1987) was used to determine the hydroxyl value.

The intrinsic viscosity of polyesters was obtained by graphical extrapolation [34].

The TGA experiments were carried out with Thermal Analyzer (STA449C) in nitrogen from 30 to $600{ }^{\circ} \mathrm{C}$ with heating rate of $10^{\circ} \mathrm{C} / \mathrm{min}^{-1}$. The measurement conditions were as follows: sample weight $5 \mathrm{mg}$, gas flow $50 \mathrm{ml} \mathrm{min}^{-1}$, and $150 \mu$ open alumina pan.

The tensile property was obtained by ZWICKI of Zwick/ Roell.

DSC experiments were carried out with 204-F1DSC of ETZSCH under nitrogen atmosphere. The sample of about $7 \mathrm{mg}$ was heated from $-100{ }^{\circ} \mathrm{C}$ to $100^{\circ} \mathrm{C}$ at $10{ }^{\circ} \mathrm{C} / \mathrm{min}^{-1}$. Then, it was cooled to room temperature.

The morphology of the coating was characterized using field emission scanning electron microscopy (SEM, JEOL JSM-6, Japan).

The hardness method was determined by marking a surface of a film with a pencil having a different hardness. The hardness test was based on the national standard GB/T 6739-1996.

The adhesion test was based on the national standard GB/T 9286-1998, which uses the cross-cut method. The

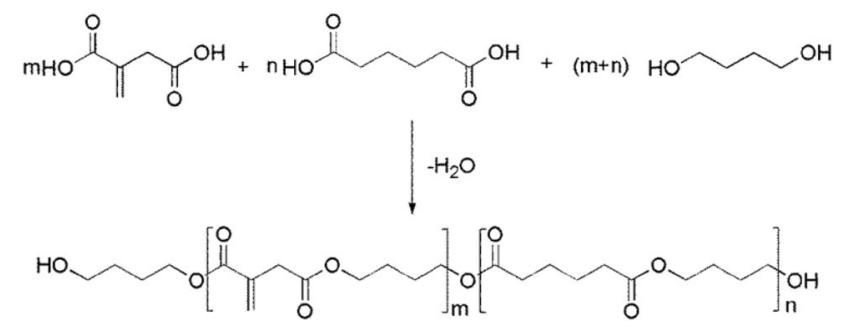

Fig. 1 The scheme of esterification

Table 1 Properties of PBIA

\begin{tabular}{llllll}
\hline Sample & $\begin{array}{l}\text { Acid value } \\
(\mathrm{mgKOH} / \mathrm{g})\end{array}$ & $\begin{array}{l}\text { hydroxyl value } \\
(\mathrm{mgKOH} / \mathrm{g})\end{array}$ & $\eta(\mathrm{dL} / \mathrm{g})$ & $\mathrm{M}_{\mathrm{w}}$ & $\mathrm{M}_{\mathrm{n}}$ \\
\hline PBIA & 40.60 & 251.72 & 0.0578 & 2023 & 1187 \\
\hline
\end{tabular}

specific experimental method was to fix the cured coating sample on a horizontal table. The cross-cut was perpendicular to the template and the cross-shaped scratches are drawn. Then the transparent tape covered the scratches evenly, and finally the transparent tape was peeled off to observe the peeling of the scratches.

The impact performance of the paint film was the ability to resist external impact. The film was rapidly deformed under high-speed gravity without the ability to crack or fall off the horseshoe patch. The impact strength test was based on the national standard GB/T1732-93.

\section{Results and discussion}

\subsection{Preparation of polyester}

In order to prepare branched chain, IA was chosen as a monomer because it had a double group. Unsaturated polyester, poly (IA-adipic acid-1,4-butanediol) (PBIA) was prepared with IA as unsaturated acid, adipic acid as saturated acid and 1,4-butanediol as dihydric alcohol, shown in Fig. 1. The molecular weight of PBIA should be low so that the polymer could have fine solubility after crosslinking. PBIA properties were shown in Table 1. The mole ratio of $-\mathrm{OH}$ and $-\mathrm{COOH}$ was 1.2:1. Hydroxyl group was excessive. So, hydroxyl value of PBI was high and PBIA molecular was low. ${ }^{1} \mathrm{H}$ NMR and FTIR of PBIA spectra were shown in Figs. 2 and 3.

In Fig. 2, The peaks at 1.697 and 4.124 ppm were attributed to the characteristic chemical shifts of protons in 1,4-butanediol. The peak at $3.372 \mathrm{ppm}$ was attributed to the characteristic chemical shifts of protons in methylene group of IA. The peaks at 5.758 and 6.361 ppm were attributed to the characteristic chemical shifts of protons in 


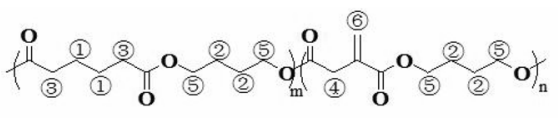

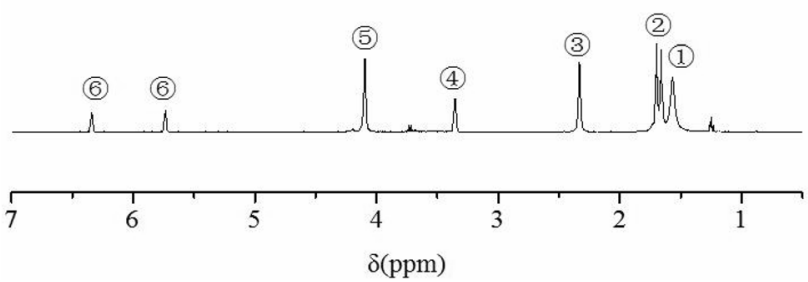

Fig. 2 1H-NMR spectrum of PBIA

double bond group of IA. The peaks at 1.57 and $2.33 \mathrm{ppm}$ were attributed to the characteristic chemical shifts of protons of adipic acid.

In Fig. 3, characteristic peak at $3547.43 \mathrm{~cm}^{-1}$ appeared due to the formation of hydroxyl group. The peaks at 2927.14 and $2853.02 \mathrm{~cm}^{-1}$ were due to the telescopic vibration absorption peak of the $\mathrm{C}-\mathrm{H}$ bond. Its bending vibration peak was 1459.69 and $1390.11 \mathrm{~cm}^{-1}$. The peak at $1734.39 \mathrm{~cm}^{-1}$ was due to $C=0$. The peaks at 1390.11 , $1179.86 \mathrm{~cm}^{-1}$ were due to $\mathrm{C}-\mathrm{O}-\mathrm{C}$, which confirmed ester group. The peak at $1639.86 \mathrm{~cm}^{-1}$ was due to $C=C$. The peaks at 955.71 and $748.29 \mathrm{~cm}^{-1}$ were due to $\mathrm{C}-\mathrm{O}-\mathrm{C}$, which confirmed ester group. These peaks exhibited the structure of PBIA.

\subsection{Crosslinking of PBIA}

PBIA could polymerize with radical initiator because it had double bonds in its molecular chain. However, crosslinking PBIA (CPBIA) solubility would decrease if its crosslinking degree was high. Therefore, the content of IA should be low. Meanwhile, the degree of crosslinking should be controlled. The content of double bond was controlled by feeding of IA during esterification. In polymerization, initiator was added with fare high dosage to ensure solubility of CPBIA with low crosslinking degree.

Molecular weight of CPBIA would increase after crosslinking. Its structure changed into crosslinking branched chain. CPBIA's properties were shown in Table 2. It was indicated that a part of $-\mathrm{OH}$ and $-\mathrm{COOH}$ esterified deeply because both acid value and hydroxyl value decreased comparing with Table 1. CPBIA's molecular weight increased for further esterification and crosslinking. So, its intrinsic viscosity increased obviously. Because of crosslinking of linear molecular structure, CPBIA's solubility changed greatly. CPBIA could dissolve in THF, ethyl acetate, acetone, methanol-toluene and ethanol-toluene. However, it could not dissolve in toluene, methanol and ethanol. Change of solubility was due to polar group $(-\mathrm{OH}$ and $-\mathrm{COOH})$ and branched chain.

The structure of CPBIA was shown in Fig. 4. FTIR spectrum of CPBIA was shown in Fig. 5. Compared with Fig. 3, the peak of $1639.86 \mathrm{~cm}^{-1}$ was very weak, which indicated that most of double bond had polymerization.

Table 2 Properties of CPBIA

\begin{tabular}{llll}
\hline Sample Acid value $(\mathrm{mgKOH} / \mathrm{g})$ & $\begin{array}{l}\text { Hydroxyl value } \\
(\mathrm{mgKOH} / \mathrm{g})\end{array}$ & $\eta(\mathrm{dL} / \mathrm{g})$ \\
\hline PBIA-C 35.40 & 187.68 & 0.0861 \\
\hline
\end{tabular}

Fig. 3 FT-IR spectrum of PBIA

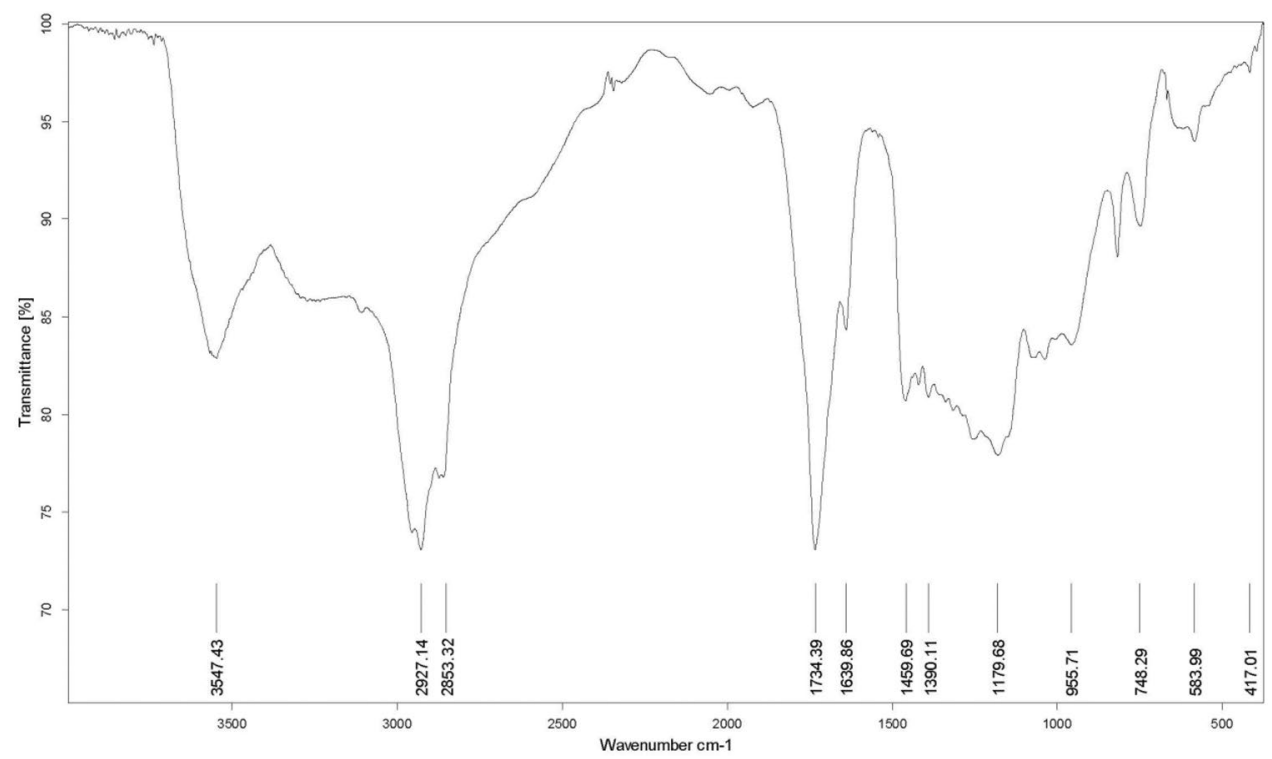




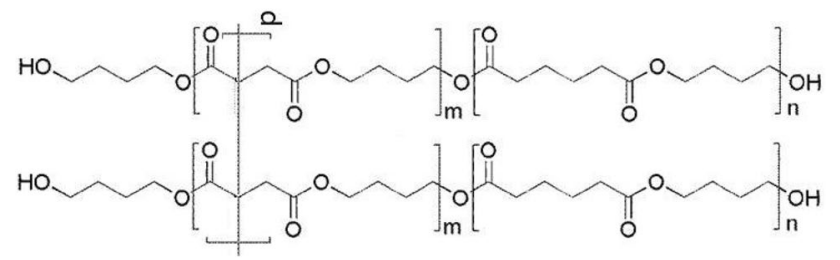

Fig. 4 The scheme of CPBIA

\subsection{Preparation of PU curing agent}

Isocyanate group of TDI could react with hydroxyl group and carboxyl group. Every molecule of CPBIA had several hydroxyl group and carboxyl group. So, the products of TDI and CPBIA would be crosslinked and insoluble. In order to ensure the solubility of curing agent, o-chlorophenol was chosen as blocking agent to react with one isocyanate group of TDI. Its blocking reaction rate was low. But its deblocking reaction rate was high and its deblocking reaction temperature was low [35]. After one isocyanate group of TDI reacted with o-chlorophenol, the activity of the rest was low. Therefore, one of isocyanate groups in TDI would be blocked by this reaction. Reaction product would have fine solubility with semi-blocked TDI because crosslinking was avoided.

New PU curing agent (TDI-CPBIA) was prepared with semi-blocked TDI and CPBIA, which FT-IR spectrum was shown in Fig. 5. The properties of PU curing agent after deblocking were shown in Table 3 (Fig. 6).

The peak of $3535 \mathrm{~cm}^{-1}$ was due to $-\mathrm{OH}$, which indicated that CPBIA was excessive. The peaks of 3330 and 1536 were due to $-\mathrm{NH}^{-\cdot}$. There is no peak of 2240-2280, which indicated that most TDI had reacted. That is to say, the concentration of free TDI was very low.

\subsection{Performance of TDI-CPBIA}

In order to investigate the properties of TDI-CPBIA, coatings were prepared based on TDI-CPBIA and poly (ethylene glycol-itaconate-succinate) (PEIS) for metal substrate. Curing temperature was $120^{\circ} \mathrm{C}$. Curing time was $2 \mathrm{~h}$.

\subsubsection{Effect of PBIA's $M_{w}$}

TDI-CPBIA was prepared with PBIA by crosslinking and reaction with TDI. So, PBIA's structure determined TDICPBIA's structure and its performance. The effect of PBIA's $M_{w}$ was studied, as shown in Table 4. PBIA's $M_{w}$ was controlled by modifying the ratio of components and polycondensation temperature. Then, CPBIA's $M_{w}$

Table 3 Properties of TDI-CPBIA

\begin{tabular}{lll}
\hline Sample & Content of $-\mathrm{NCO}$ & $\eta(\mathrm{dL} / \mathrm{g})$ \\
\hline PU curing agent & $2.04 \%$ & 0.1 \\
\hline
\end{tabular}

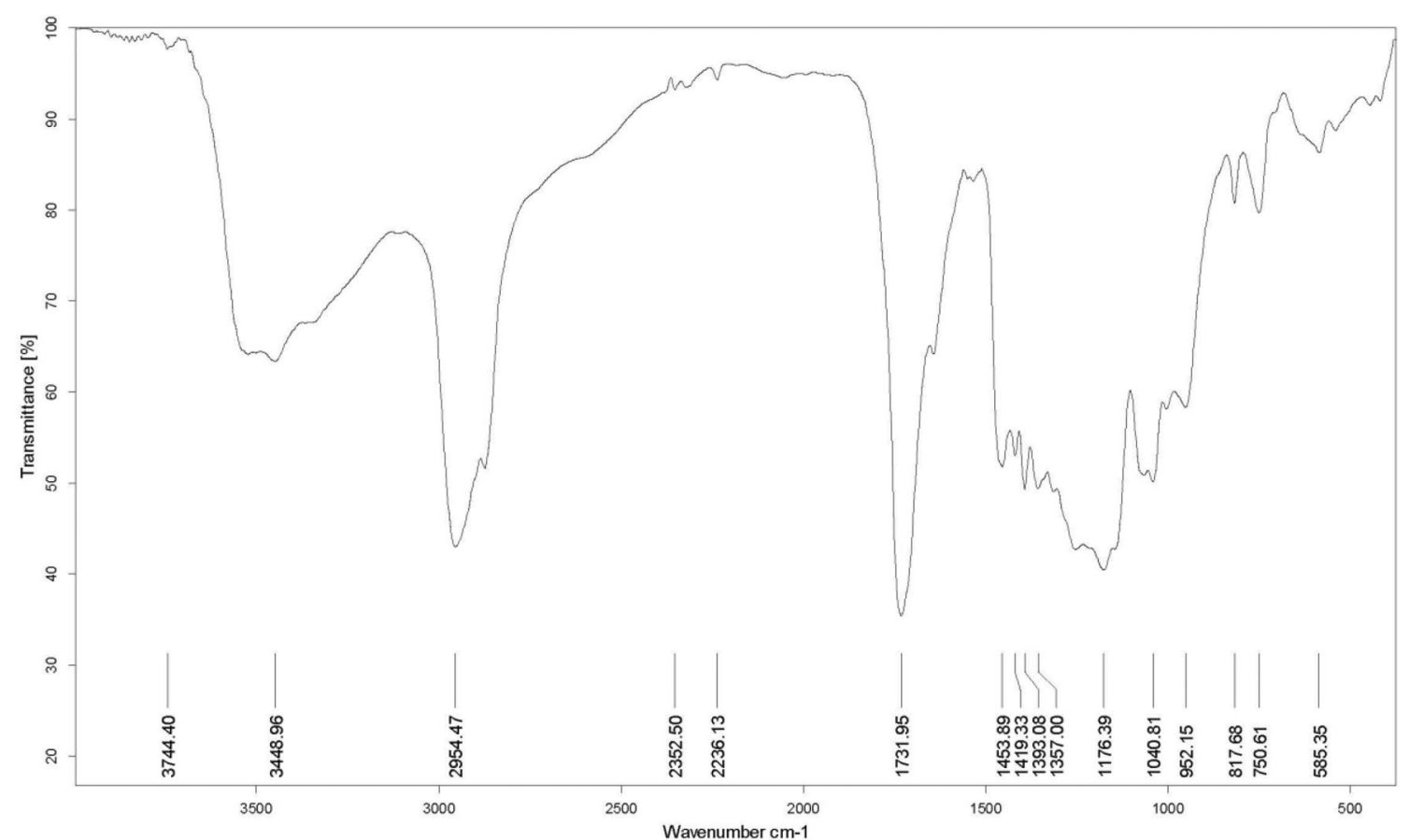

Fig. 5 FT-IR spectrum of CPBIA 


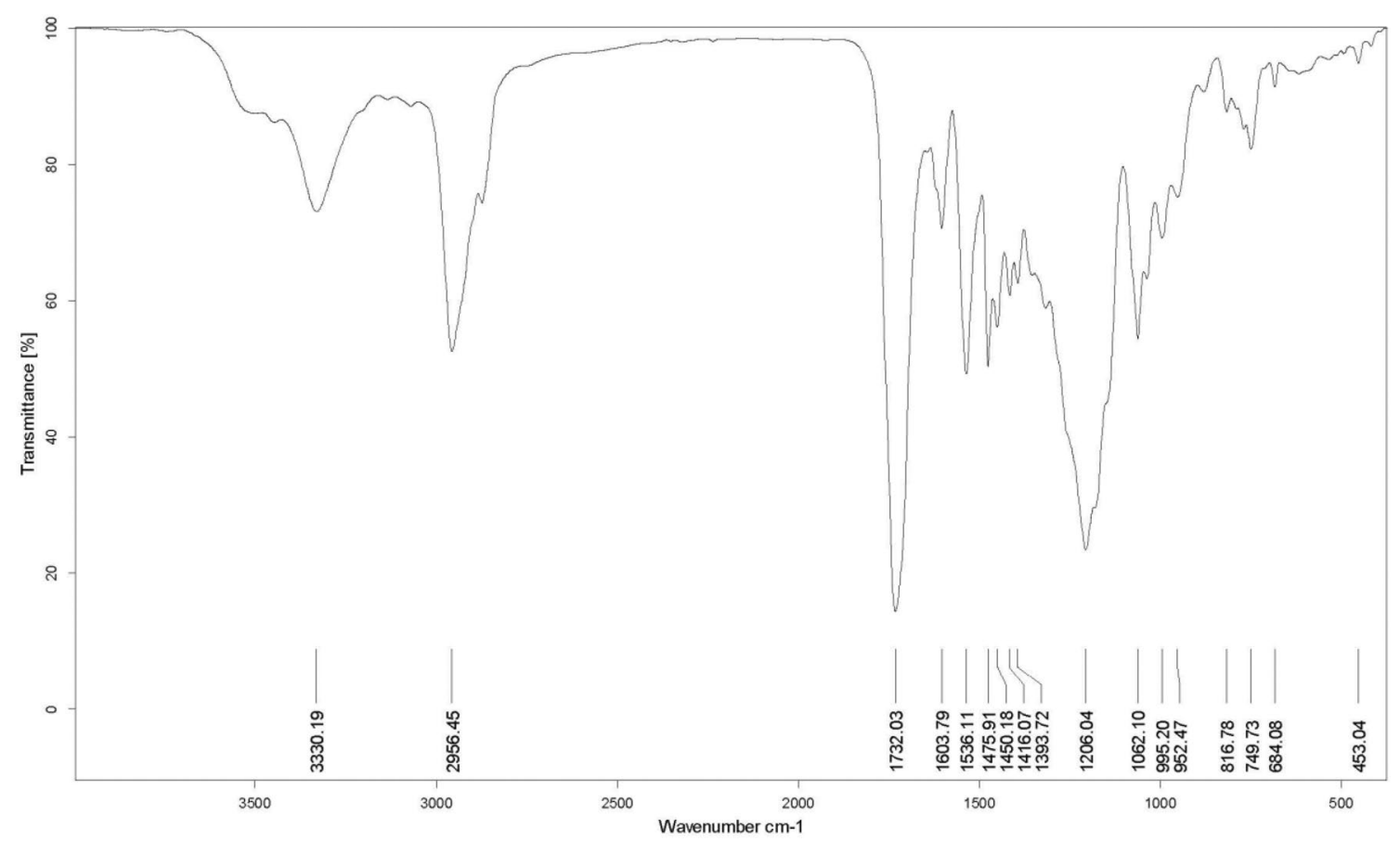

Fig. 6 FT-IR spectrum of TDI-CPBIA

Table 4 Effect of PBIA's $M_{w}$ on the properties of coating

\begin{tabular}{lllll}
\hline PBIA's $M_{w}$ & $\begin{array}{l}\text { Pencil } \\
\text { hardness } \\
(H)\end{array}$ & $\begin{array}{l}\text { Adhesion (cross } \\
\left.\text { cut test, } 1 \mathrm{~mm}^{2}\right)\end{array}$ & $\begin{array}{l}\text { Impact test } \\
(500 \mathrm{~g}, \mathrm{~cm})\end{array}$ & $\begin{array}{l}\text { Coating } \\
\text { thickness } \\
(\mu \mathrm{m})\end{array}$ \\
\hline 1309 & 4 & 1 & $>50$ & 49 \\
1678 & 5 & 1 & $>50$ & 48 \\
2023 & 6 & 1 & $>50$ & 48 \\
2301 & 6 & 2 & $>50$ & 49 \\
\hline
\end{tabular}

was controlled by the dosage of initiator and polymerization temperature.

With increasing of PBIA's $\mathrm{M}_{\mathrm{w}^{\prime}}$ TDI-CPBIA had high molecular weight accordingly. After curing with polyether polyol, the degree of crosslinking would be higher. So, the hardness of the coating increased, as shown in Table 4. In term of adhesion, the performance was low when PBIA's Mw was the highest 2301. With increasing of PBIA's $M_{w}$, the branches were closer and interacted each other, which limited the branch activity. The force between coating and base material decreased accordingly. All sample performed good in impact test. In general, TDI-CPBIA was a good PU curing agent.

\subsubsection{Thermal performance of the coating}

Thermal performance of the coating was shown in Fig. 7 and Table 5 with different $\mathrm{NCO} / \mathrm{OH}$ ratio. It was shown that $\mathrm{T}_{0}$ and $\mathrm{T}_{\mathrm{f}}$ of four sample were same, as about $255^{\circ} \mathrm{C}$ and $485^{\circ} \mathrm{C}$, respectively. It indicated that these products had same structure. The difference was the temperature at different mass loss. When mass loss was lower than $50 \%$, the curves were same. However, the ratio of mass loss decreased with $\mathrm{NCO} / \mathrm{OH}$ ratio increasing when mass loss was higher than $50 \%$. More NCO group would react with $\mathrm{H}_{2} \mathrm{O}$ with high $\mathrm{NCO} / \mathrm{OH}$ ratio. So, crosslinking degree between molecular chain increased. Therefore, the mass loss decreased.

PU coating had three types of thermal decomposition, which was affected by molecular crosslinking, hydrogen bond and inner crystalline structure. Firstly, $\mathrm{C}-\mathrm{O}$ and $\mathrm{C}-\mathrm{N}$ bonds of PU coating were broken into isocyanate and polyol under high temperature. Secondly, carbamate was broken into primary amine, alkene and $\mathrm{CO}_{2}$. Thirdly, elimination reaction of carbamate occurred to form secondary amine and $\mathrm{CO}_{2}$. Reaction mechanism was shown in Fig. 8 $[36,37]$.

In DTG of PEIS-PU coating, there were obviously four temperature peaks, which corresponded to the temperature of maximum mass-loss rate. The first peak was at about $286^{\circ} \mathrm{C}$, which corresponded to the decomposition of ester bond in carbamic acid ester of PEIS-IU. The highest peak was at about $390^{\circ} \mathrm{C}$ between $320^{\circ} \mathrm{C}$ and $450^{\circ} \mathrm{C}$, which corresponded to decomposition of polyester segment. In this area, mass loss rate increased with increasing of polyester content. The last mass loss area was at $450 \sim 500^{\circ} \mathrm{C}$, which corresponded to isocyanate group. In 

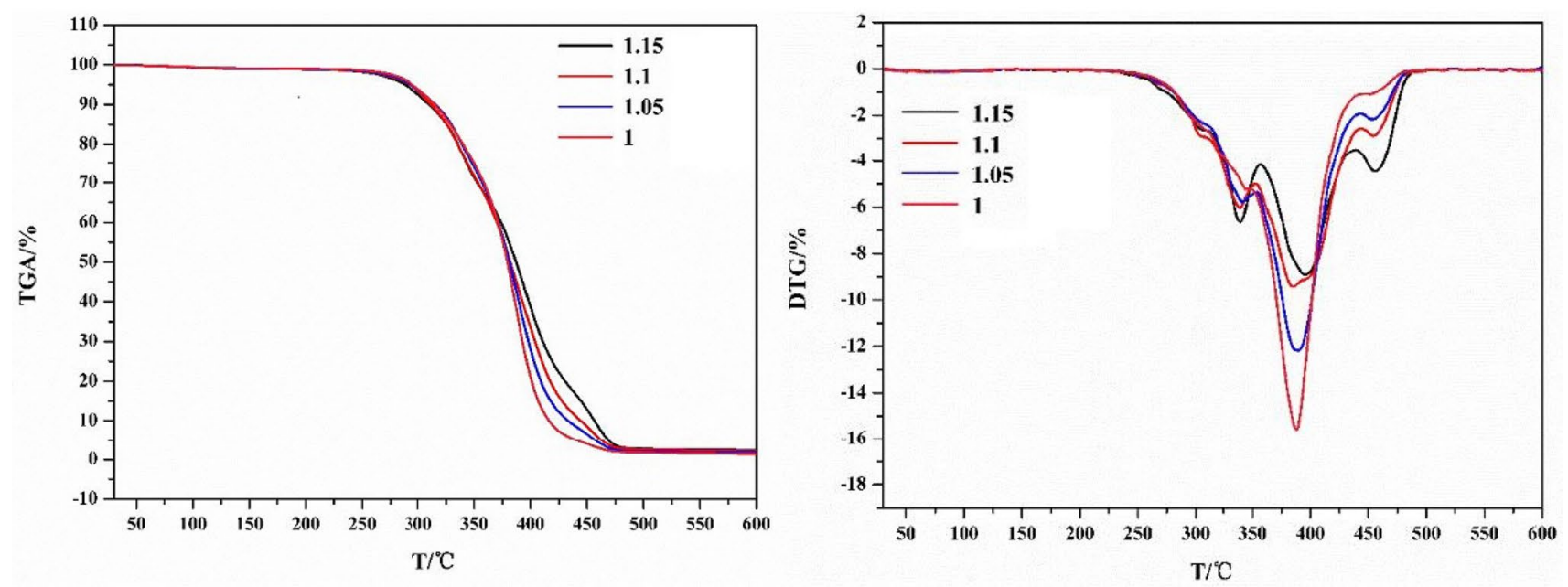

Fig. 7 TG of PEIS-PU with several $\mathrm{NCO} / \mathrm{OH}$ ratio

Table 5 Thermal analysis of PEIS-PU

\begin{tabular}{lllllll}
\hline NCO/OH ratio & $\mathrm{T}_{0} /{ }^{\circ} \mathrm{C}$ & $\mathrm{T}_{0.1} /{ }^{\circ} \mathrm{C}$ & $\mathrm{T}_{0.2} /{ }^{\circ} \mathrm{C}$ & $\mathrm{T}_{0.5} /{ }^{\circ} \mathrm{C}$ & $\mathrm{T}_{0.8} /{ }^{\circ} \mathrm{C}$ & $\mathrm{T}_{\mathrm{f}} /{ }^{\circ} \mathrm{C}$ \\
\hline 1.15 & 258 & 305 & 335 & 386 & 430 & 484 \\
1.1 & 255 & 311 & 355 & 382 & 417 & 488 \\
1.05 & 256 & 315 & 339 & 381 & 408 & 489 \\
1 & 254 & 314 & 339 & 379 & 400 & 483 \\
\hline
\end{tabular}

The numbers of $0.1,0.2,0.5$ and 0.8 were mass loss of $10,20,50$ and $80 \%$, respectively

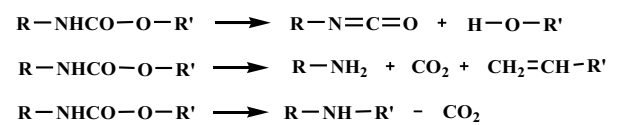

Fig. 8 The mechanism of thermal decomposition of PU coating

conclusion, coating thermostability would be better with high molar ratio to $\mathrm{NCO} / \mathrm{OH}$ and low content of polyester.

\subsubsection{Physical properties of the coating}

Physical properties of the coating would be different with different ratio of two components in two component polyurethane. If the component $\mathrm{A}$ ( $\mathrm{PU}$ curing agent) was too little, some hydroxyl group would remain. The content of carbamic acid ester was little. In the coating, there were a lot of hydroxyl group, which was hydrophilic. Therefore, the coating was soft and low-hydrolytic resistance. On the contrary, excess isocyanate group would react with $\mathrm{H}_{2} \mathrm{O}$ to form carbamide group with high content of component A. Crosslinking degree would increase, which made the coating fragile and low impact resistant. Optimized ratio of $\mathrm{NCO} / \mathrm{OH}$ was about $1 \sim 1.15$ with fine coating performance.
Coating performance was shown in Table 6 with different $\mathrm{NCO} / \mathrm{OH}$ molar ratio. It was shown that it was high solid content coating (above $80 \%$ ) so that low organic solvent would be needed. With the decrease of NCO/ $\mathrm{OH}$ molar ratio, surface drying time and hard drying time increased and coating hardness decreased. Impact strength increased, too. Excessive NCO group would react with $\mathrm{H}_{2} \mathrm{O}$ and release a lot of heat which accelerate solvent evaporation. So, drying time decreased with high NCO/ $\mathrm{OH}$ molar ratio. Meanwhile, there were more crosslinking among coating molecule with the result of high hardness and fragile. Coating impact strength decreased with harder segment. In addition, the coating of PEIS and CPBIA had a fare drawback that it would shrink a little during curing. Heat release would be fast because NCO group of CPBIA had high reactivity. High solvent evaporation resulted in low levelling. Meanwhile, polyester had high surface tension which caused low wettability. So, the coating would shrink a little.

Mechanical properties of the coating were studied with PEIS and polyether polyol (DL2000D) as the component $B$. The coatings were prepared with PEIS and CPBIA (PEISPU) or DL2000D and CPBIA (DL2000D-PU). The results were shown in Figs. 9 and 10 with different component $\mathrm{A} / \mathrm{B}$ ratio. When $\mathrm{NCO} / \mathrm{OH}$ molar ratio decreased, Rigid 
Table 6 Coating performance with different $\mathrm{NCO} / \mathrm{OH}$ mole ratio

\begin{tabular}{lllllll}
\hline $\begin{array}{l}\text { NCO/OH } \\
\text { molar ratio }\end{array}$ & Mass content /\% & $\begin{array}{l}\text { Surface dry- } \\
\text { ing time } / \mathrm{h}\end{array}$ & $\begin{array}{l}\text { Hard dry- } \\
\text { ing time } / \mathrm{h}\end{array}$ & $\begin{array}{l}\text { Pencil } \\
\text { hardness } \\
(\mathrm{H})\end{array}$ & $\begin{array}{l}\text { Adhesion (cross } \\
\left.\text { cut test, } 1 \mathrm{~mm}^{2}\right)\end{array}$ & $\begin{array}{l}\text { Impact test } \\
(500 \mathrm{~g}, \mathrm{~cm})\end{array}$ \\
\hline 1.15 & 82.46 & 1.2 & 12 & 4 & 3 & 35 \\
1.1 & 86.96 & 1.5 & 10 & 4 & 3 & 45 \\
1.05 & 88.81 & 1.8 & 15 & 3 & 3 & 45 \\
1 & 90 & 2 & 23 & 3 & 4 & 43 \\
\hline
\end{tabular}

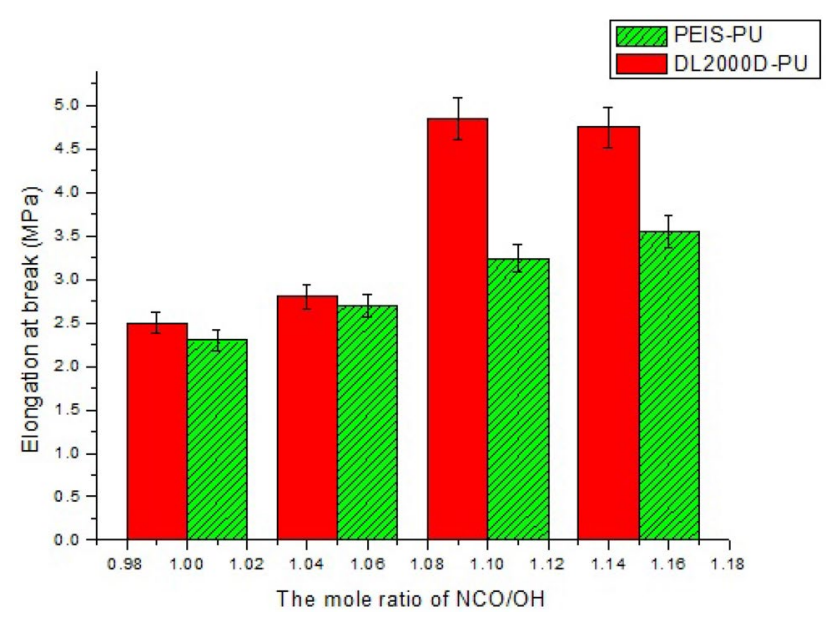

Fig. 9 Tensile strength of the coating with PEIS-PU and DL2000DPU

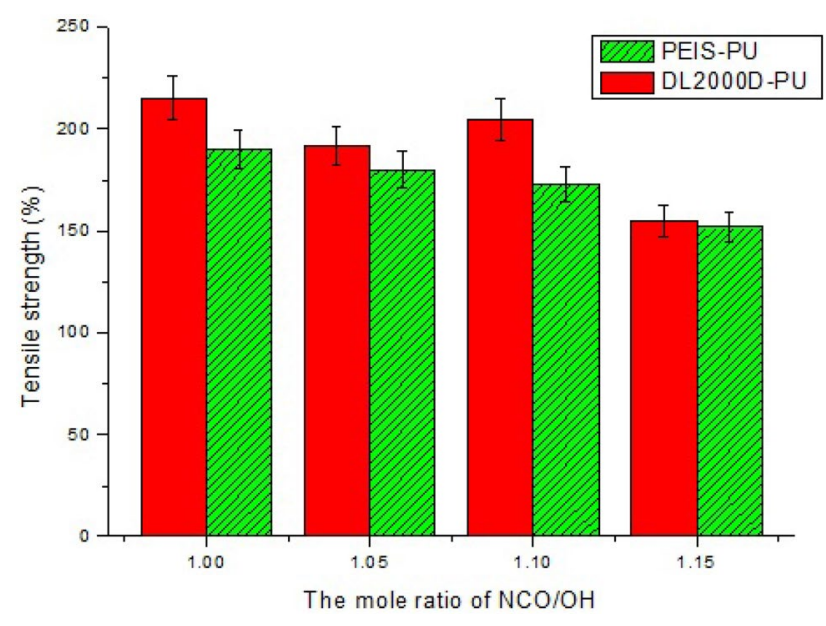

Fig. 10 Elongation at break PEIS-PU and DL2000D-PU

chain of carbamate decreased and soft chain of aliphatic chain and ether group increased. Meanwhile, crosslinking degree would increase with high $\mathrm{NCO} / \mathrm{OH}$ molar ratio. Because of increasing of soft chain in component $B$ and crosslinking degree decreased, the coating chain was movable and rotatable. So, tensile strength decreased as shown in Fig. 9 and coating elongation increased as shown in Fig. 10. Comparing to PEIS, the ether group of DL2000L was more rotatable. So, with increasing of the component $B$, coating tensile strength decreased fast with DL2000D.

\subsubsection{SEM of the coating}

The images of several coating fracture surface were shown in Figs. 11 and 12. It was shown that there was no tiny bubble and coating stripping and it was smooth. It was indicated that TDI-CPBIA had fine intermiscibility with PBIA and DL2000D. Two components could mix uniformly. That was the reason that the coating had good tensile strength and elongation. In Fig. 12a, there was a fissure in the coating. $\mathrm{NCO} / \mathrm{OH}$ molar ratio was 1.15 for this sample. The content of NCO group was high. And that, TDI-CPBIA had branched chain. Most terminal group was -NCO. The crosslinking degree was high. During curing, the reaction between $-\mathrm{NCO}$ and $-\mathrm{OH}$ was fast. More heat was released and solvent evaporation of was fast. After the coating became solid, there was a little solvent evaporating. So, the coating shrinked and breaked.

\section{Conclusions}

A PU curing agent with new structure was designed, which was branched and low toxicity. These polymers were characterized by several methods, such as ${ }^{1} \mathrm{H}$ NMR, GPC, FT-IR and so on. $\mathrm{M}_{\mathrm{w}^{\prime}}$ acid value and hydroxyl value of PBIA were about 2000, 40 and $250 \mathrm{mgKOH} / \mathrm{g}$, respectively. The peaks of the characteristic chemical shifts of protons in 1,4-butanediol, IA and adipic acid occurred in the ${ }^{1} \mathrm{H}$-NMR and FT-IR spectra. It was indicated that the structure of polymer was same as designed. These polymers had suitable molecular weight and solubility.

After radical polymerization, acid value and hydroxyl value of PBIA changed into 35.4 and 187.68, respectively. It was indicated that a little of acid and hydroxyl group reacted again. The peak of double bond was very weak in FT-IR spectrum. The intrinsic viscosity changed from 0.0578 to $0.0861 \mathrm{dL} / \mathrm{g}$. PBIA was crosslinked under controllable degree. 
Fig. 11 SEM images of PBIA-PU coating with different $\mathrm{NCO} /$ $\mathrm{OH}$ molar ratio (a 1.15 ; b 1.1 ; c $1.05, \mathbf{d} 1$ )

Fig. 12 SEM images of DL2000D-PU coating with $\mathrm{NCO} / \mathrm{OH}$ molar ratio $(\mathbf{a} 1.15 ; \mathbf{b}$ $1.1 ; \mathbf{c} 1.05, \mathbf{d} 1$ )
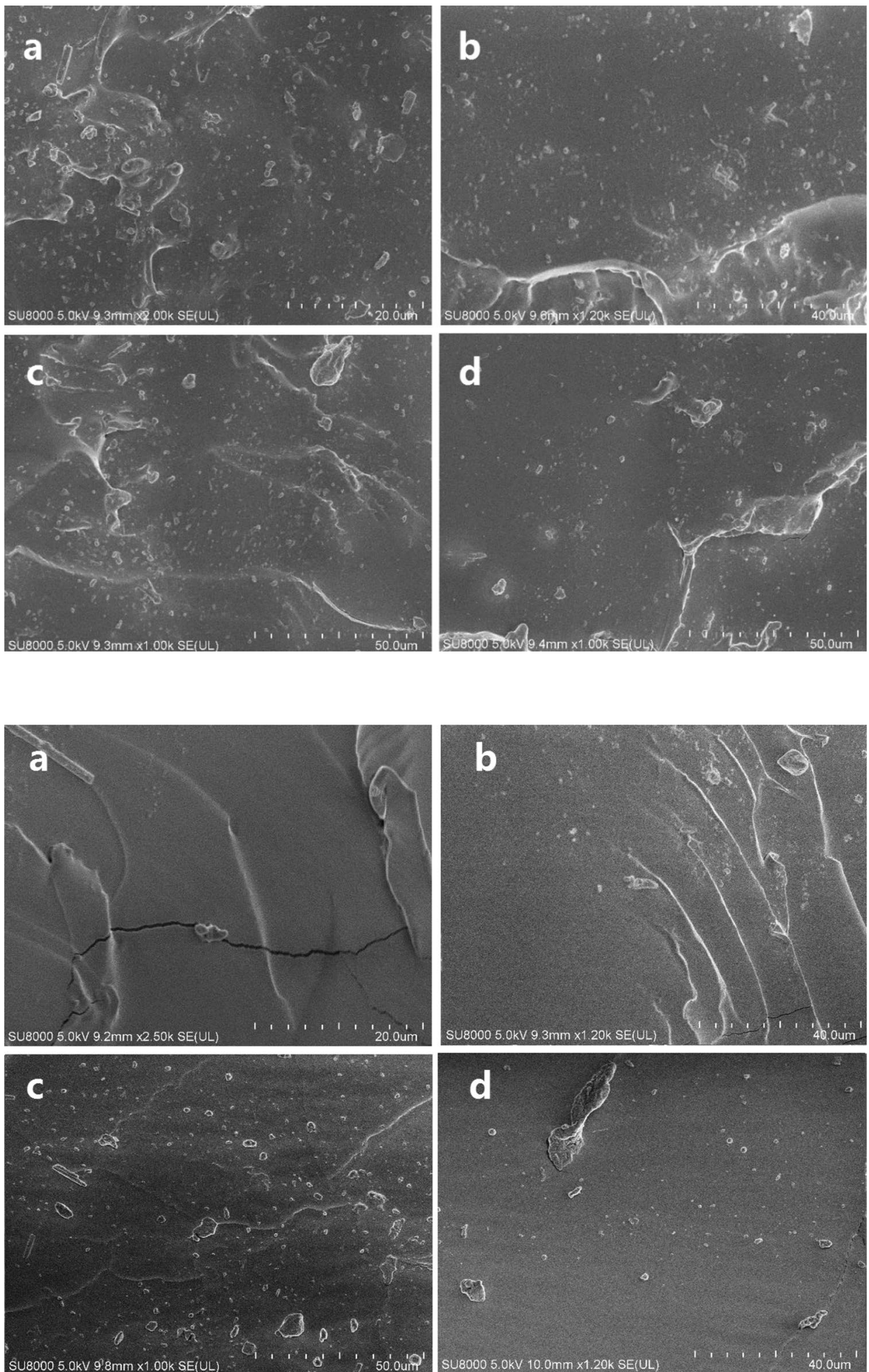

It was indicated in FT-IR spectrum of TDI-CPBIA that the concentration of free TDI was very low. The new PU curing agent had a suitable content of -NCO.

TDI-CPBIA had fine application performance. Pencil hardness, adhesion and impact test were above $6 \mathrm{H}$, level
1 and $50 \mathrm{~cm}$. Meanwhile, the PEIS-PU had good thermal performance with $\mathrm{T}_{0}>25^{\circ} \mathrm{C}$. It was shown in SEM images that there was no tiny bubble and coating stripping and it was smooth. TDI-CPBIA had fine intermiscibility with PBIA and DL2000D. 
Acknowledgements This work is supported by Open Fund of Shandong Key Laboratory of Corrosion Science (KLCS201911) and Qingdao Post-Doctoral Fund (Y4KY14110N).

\section{Compliance with ethical standards}

Conflict of interest On behalf of all authors, the corresponding author states that there is no conflict of interest.

\section{References}

1. Madbouly SA, Otaigbe JU (2009) Recent advances in synthesis, characterization and rheological properties of polyurethanes and POSS/polyurethane nanocomposites dispersions and films. Prog Polym Sci 34:1283-1332

2. Parmar R, Patel K, Parmar J (2005) High-performance waterborne coatings based on epoxy-acrylic-graft-copolymer-modified polyurethane dispersions. Polym Int 54:488-494

3. Naik RB, Ratna D, Singh SK (2014) Synthesis and characterization of novel hyperbranched alkyd and isocyanate trimer based high solid polyurethane coatings. Prog Org Coat 77:369-379

4. Patel KI, Parmar RJ, Parmar JS (2008) Novel binder system for ultraviolet-curable coatings based on tobacco seed (Nicotiana rustica) oil derivatives as a renewable resource. J Appl Polym Sci 107:71-81

5. Cui J, Zhao M, Wang HB, Xiao-Dong LI (2017) Study and application of UV-curable coatings. Mod Paint Finish 20:20-22

6. Johansson M, Malmström E, Jansson A, Hult A (2000) Novel concept for low temperature curing powder coatings based on hyperbranched polyesters. J Coat Technol 72:49-54

7. Raychura AJ, Jauhari S, Prajapati VS, Dholakiya BZ (2018) Synthesis and performance evaluation of vegetable oil based wood finish polyurethane coating research article. Bioresour Technol Rep 3:88-94

8. He R, Wang J, Wang X, Li W, Zhang X (2018) Fabrication and characterization of core-shell novel PU Microcapsule using TDI trimer for release system. Colloids Surf A 550:138-144

9. Bello A, Xue Y, Gore R, Woskie S, Bello D (2019) Assessment and control of exposures to polymeric methylene diphenyl diisocyanate (pMDI) in spray polyurethane foam applicators. Int J Hyg Environ Health 222:804-815

10. Xiao Y, Zhao H, Wu B, Fu X, Hu K, Zhou C, Lei J (2016) Preparation and characterization of waterborne polyurethane based on diphenylmethane diisocyanate-50. Adv Polym Technol 37:21700

11. Loshini SD, Adhikari Raju, Bown Mark, Shanks Robert, Benu A (2019) Morphology and surface properties of high strength siloxane poly(urethane-urea)s developed for heart valve application. J Biomed Mater Res Part B Appl Biomater 107:112-121

12. Rasl NA, Zubir SA (2020) Synthesis and characterization of IPDI based shape memory polyurethane. Mater Sci Forum 5946:142-147

13. Ortega FJ, Roman C, Navarro FJ, García-Morales M, Mcnally T (2016) Physico-chemistry control of the linear viscoelastic behaviour of bitumen/montmorillonite/MDI ternary composites: effect of the modification sequence. Fuel Process Technol 143:195-203

14. Zheng LL, Xie HL, Wu YL, Huang JH (2012) Study on synthesis of environmental waterborne polyurethane. Adv Mater Res 550-553:653-656

15. Davletbaeva IM, Zaripov II, Karimullin RR, Gumerov AM, Davletbaev RS, Sharifullin RR, Parfenov VV (2017) Framed aromatic polyurethanes based on an anionic macroinitiator, 4,4"-diphenylmethane diisocyanate, and 4,4"-dihydroxy2,2-diphenylpropane: Synthesis and characterization. Polym Sci 59:43-50

16. Arnold SM, Collins MA, Graham C, Jolly AT, Parod RJ, Poole A, Schupp T, Shiotsuka RN, Woolhiser MR (2012) Risk assessment for consumer exposure to toluene diisocyanate (TDI) derived from polyurethane flexible foam. Regul Toxicol Pharmacol 64:504-515

17. He Y, Zhang X, Zhang X, Huang H, Chang J, Chen H (2012) Structural investigations of toluene diisocyanate (TDI) and trimethylolpropane (TMP)-based polyurethane prepolymer. J Ind Eng Chem 18:1620-1627

18. Gagné S, Lesage J, Ostiguy C, Tra HV (2003) Determination of unreacted 2,4-toluene diisocyanate (2,4TDI) and 2,6-toluene diisocyanate $(2,6 \mathrm{TDI})$ in foams at ultratrace level by using HPLC-CIS-MS-MS. Anal 128:1447-1451

19. Ochiai T, Muneyasu M, Sasaki K, Hinamoto T (2001) Experimental method for evaluating isocyanate conversion and trimer formation in polyisocyanate-polyurethane foams. Eur Polym J 37:949-954

20. Marans N, Gluecksmann A (1979) Removal of unreacted tolylene diisocyanate from urethane prepolymers, US, 4169175

21. Lutišan J, Cvengroš J, Micov M (2002) Heat and mass transfer in the evaporating film of a molecular evaporator. Chem Eng J 85:225-234

22. Robin J, Blind A (1989) Substantially pure isocyanurate/polyisocyanates, US, 4864025

23. Wilhelm B (1966) Glycol modified isocyanurate containing polyisocyanates, US, 3248372

24. Milligan B, Starner WE, Grandin RE, Casey JP (1987) Toluènediisocyanates à faible point de fusion contenant des groupements urethanes, EP, 0252425

25. Starner WE, Toseland BA, Machado RM (1993) Preparation of urethane prepolymers having low levels of residual diisocyanate, EP, 0420026

26. Schnabel WJ, O'Connor JM (1983) Removal of unreacted diisocyanate from polyurethane prepolymers, US, 4385171

27. Li B, Cui X, O'Nolan D, Wen HM, Jiang M, Krishna R, Wu H, Lin RB, Chen YS, Yuan D (2017) An ideal molecular sieve for acetylene removal from ethylene with record selectivity and productivity. Adv Mater 29:1704210

28. Jie Z, Yongji W, Wenying Z, Zhiqiang C (2002) Study on the ways reducing the content of free TDI. Polyurethane Ind 17:10-13

29. Subramani S, Park YJ, Lee YS, Kim JH (2003) New development of polyurethane dispersion derived from blocked aromatic diisocyanate. Prog Org Coat 48:71-79

30. Heiss HL (1968) Prepolymer composition, US, 3384624

31. Alois G (1965) Isocyanates and method of preparing same, US, 3183112

32. Jackle WA (1980) Polyether based urethanes with superior dynamic properties, US, 4182825

33. Siuta AJ, Starner WE, Toseland BA (1993) Preparation of urethane prepolymers having low levels of residual diisocyanate, $E P, 0420026$

34. Abdel-Azim AAA, Atta AM, Farahat MS, Boutros WY (1998) Determination of intrinsic viscosity of polymeric compounds through a single specific viscosity measurement. Polymer 39:6827-6833

35. Yang PF, Han YD, Li TD (2011) Effect of Substituents and Solvents on Phenol-Isocyanate Urethane Reaction. Adv Mater Res 150-151:23-26

36. Molero C, Lucas AD, Rodríguez JF (2008) Recovery of polyols from flexible polyurethane foam by "split-phase" glycolysis: 
Study on the influence of reaction parameters. Polym Degrad Stab 93:353-361

37. Ravey M, Pearce EM (2015) Flexible polyurethane foam. I. thermal decomposition of a polyether-based, water-blown commercial type of flexible polyurethane foam. J Appl Polym Sci 63:47-74
Publisher's Note Springer Nature remains neutral with regard to jurisdictional claims in published maps and institutional affiliations. 